early stent thrombosis was observed in Group A. The 30-day death rate was $6 \%$ with $2.6 \%$ in Group A.

Conclusions Use of bivalirudin and prasugrel in the acute treatment of STEMI demonstrates excellent efficacy and safety. The HorizonsAMI data suggested a risk of early stent thrombosis in the absence of heparin, however, our heparin-naive cohort were free of this complication. Furthermore, our combined strategy of anti-thrombotic therapy and preferred radial access maintains very low accesssite complication rates. Randomised trial data confirming this strategy is awaited.

\section{CORONARY WAVE INTENSITY: A NOVEL INVASIVE TOOL FOR PREDICTING MYOCARDIAL VIABILITY FOLLOWING ACUTE CORONARY SYNDROMES}

doi:10.1136/heartjnl-2012-301877b.133

K De Silva, ${ }^{*}$ A Guilcher, T Lockie, M Marber, S Redwood, S Plein, D Perera. St. Thomas' Hospital/King's College London, London, UK

Introduction Wave intensity analysis (WIA) uses simultaneous changes in intracoronary pressure and flow to characterise energy transfer within the coronary circulation. In normal hearts, flow is predominantly driven by a microcirculatory-derived, diastolic phase, backward expansion wave (BEW) and aortic-derived, systolic phase, forward compression wave (FCW) (Abstract 133 figure 1A). Regional changes in contraction and microvascular function following acute coronary syndromes (ACS) may affect these waves, but the utility of WIA in this setting remains unknown.

Methods Patients were included 2-7 days after presenting with Non-ST elevation myocardial infarction (NSTEMI). Those with prior MI, haemodynamic instability or coronary artery disease unsuitable for PCI were excluded. Left ventricular ejection fraction (LVEF) and late-gadolinium enhancement (LGE) were assessed by cardiac MRI. Subsequently, intra-coronary (IC) pressure and Doppler measurements were taken in the infarct-related artery (IRA) and a remote reference vessel (REF), during IC adenosineinduced hyperaemia. Blinded WIA was performed offline. Regional left ventricular recovery following percutaneous coronary intervention (PCI) was quantitatively assessed by MRI, at 3 months (Abstract 133 figure 1B). Pearson Regression analysis was performed to assess the statistical relationship between WIA and size of infarction and recovery in function, following PCI.

Results 18 patients $(57 \pm 11 \mathrm{yrs}) 88 \pm 51 \mathrm{~h}$ post-myocardial infarction were enrolled. 12-h Troponin T, LVEF and \% left ventricular infarct (LGE) mass were $1.53 \pm 1.40 \mu \mathrm{g} / 1,56 \% \pm 11.1 \%$ and $8.9 \% \pm 6.0 \%$ respectively. BEW and FCW energies predominated with the mean peak WI being -3.17 and $+2.80\left(\mathrm{~W} / \mathrm{m}^{2} / \mathrm{s}^{2} \times 10^{5}\right)$ respectively. IRA BEW energy strongly correlated with regional left ventricular recovery $\left(\mathrm{R}^{2}=0.53, \mathrm{p}=0.005\right)$ and was inversely correlated to infarct mass $\left(R^{2}=0.63, p<0.0001\right)$ (Abstract 133 figure $\left.1 C\right)$. REF BEW correlated weakly with LGE $\left(\mathrm{R}^{2}=0.34, \mathrm{p}=0.03\right)$.

Conclusion The microcirculatory backward expansion wave energy correlates with the magnitude and location of infarction and is

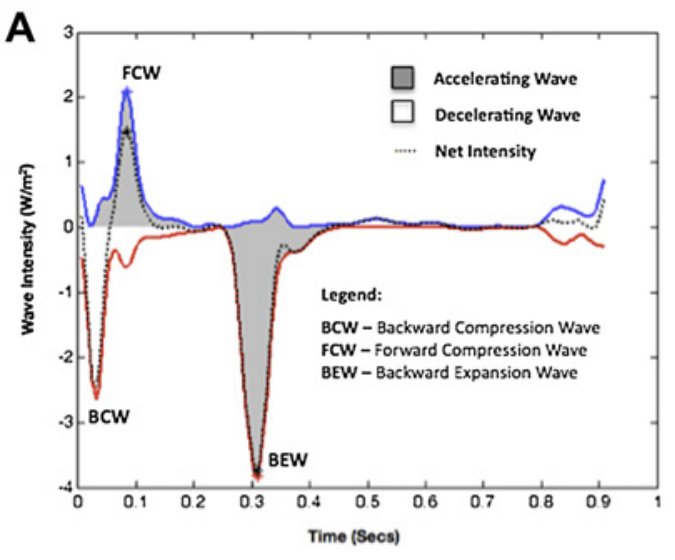

B

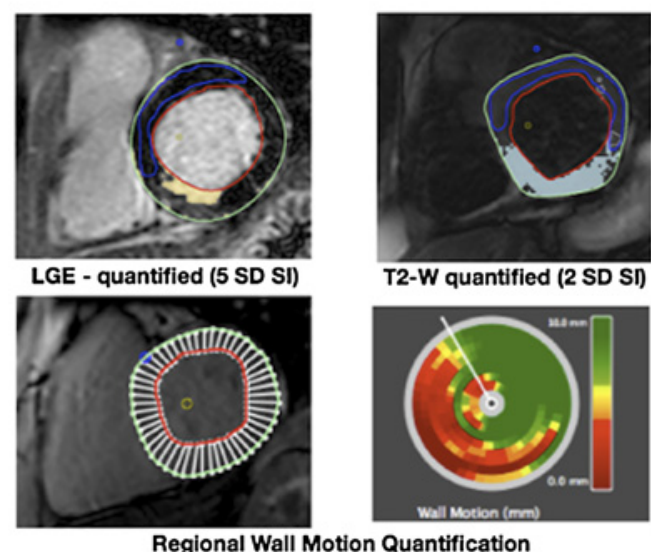

Abstract 133 Figure 1 (A) Typical Coronary Wave Intensity Profile. (B) Quantitative LGE Mass and Regional Wall Motion Assessment. (C) Peak IRA BEW Wave Intensity vs LGE Mass. (D) BEW Correlation with Regional LV Recovery. 
predictive of recovery in function following acute coronary syndromes. Therefore, coronary wave intensity analysis may be a useful adjunctive tool during cardiac catheterisation in the assessment of viability following ACS.

\section{THE ROLE OF CARDIOVASCULAR MRI IN TROPONIN POSITIVE ACUTE CORONARY SYNDROMES WITH UNOBSTRUCTED CORONARY ARTERIES}

doi:10.1136/heartjnl-2012-301877b.134

E McAlindon, * A G Porto, A Chiarellli, G Cincotta, C Lawton, C Bucciarelli-Ducci. Bristol Heart Institute, Bristol, UK

Background Patients with acute coronary syndrome and unobstructed coronary arteries represent a clinical dilemma in whom clinical management is uncertain. Cardiovascular magnetic resonance (CMR) has the potential to non-invasively identify the presence of myocardial infarction or acute myocarditis, thus establishing a final diagnosis with management implications.

Aim To assess the diagnostic value of CMR in patients presenting with ACS and unobstructed coronary arteries.

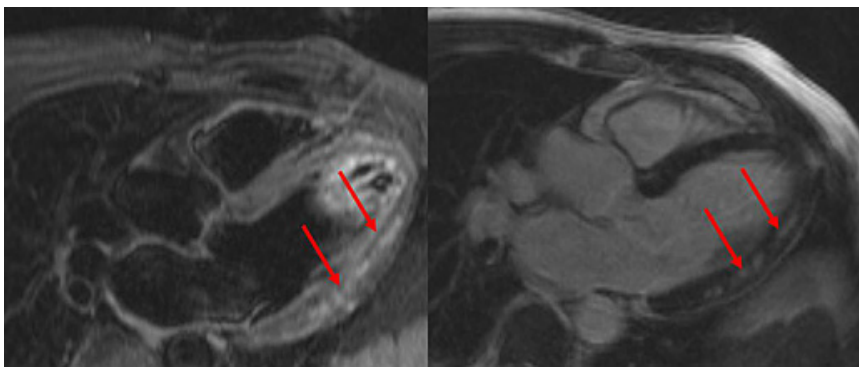

Abstract 134 Figure 1 Acute myocarditis with mid myocardial and epicardial oedema and fibrosis.

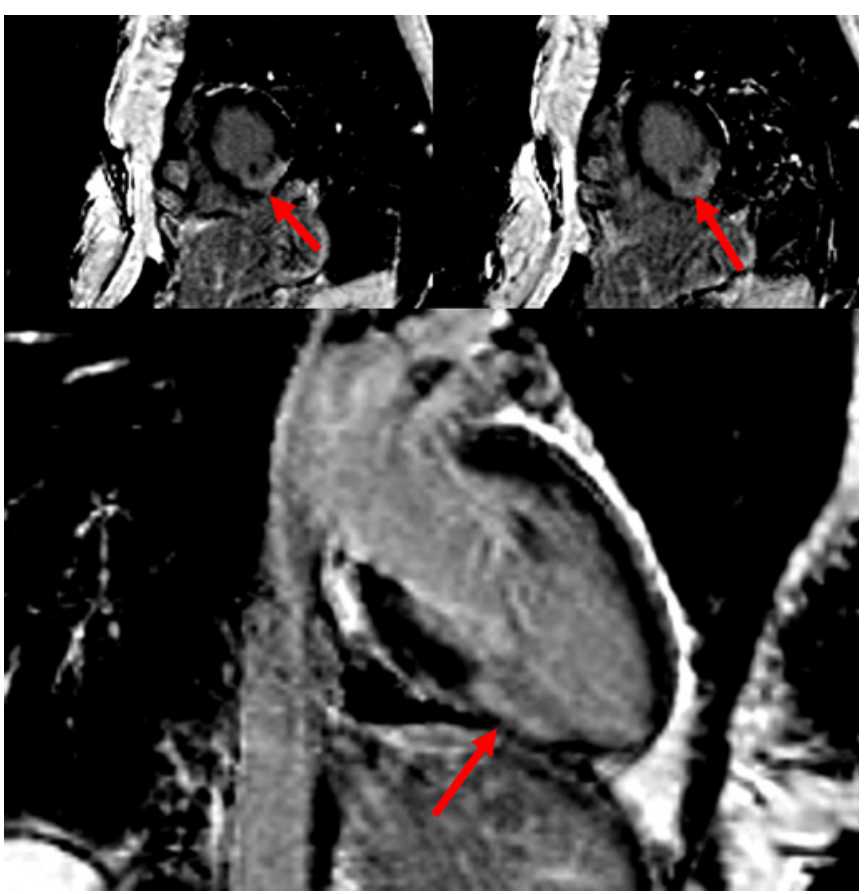

Abstract 134 Figure 2 Acute myocardial infarction with late gadolinium enhancement in the mid-cavity and apical inferior walls.
Methods From October 2010 to November 2011, 48 patients who presented with troponin positive ACS and unobstructed coronary arteries were consecutively recruited. A comprehensive CMR protocol, including T2 weighted STIR imaging for oedema and late gadolinium enhancement imaging for myocardial scarring, was performed within 4 weeks of the index event.

Results In $75 \%$ of cases, a cause for the troponin rise was found. Based on the oedema and scarring patterns observed, the most common diagnoses were acute myocarditis (Abstract 134 figure 1) in $40 \%$ of cases and acute myocardial infarction with spontaneous coronary recanalisation/embolus (Abstract 134 figure 2) in 19\% of the cases. In six patients (12\%) a diagnosis of cardiomyopathy was established: dilated cardiomyopathy $(n=3)$, hypertrophic cardiomyopathy $(n=1)$ and Tako-Tsubo cardiomyopathy $(n=2)$. Acute pericarditis was present in two patients (4\%). The remaining $25 \%$ of patients had a normal CMR scan.

Conclusion In the setting of acute coronary syndromes with unobstructed coronary arteries CMR was able to establish a final diagnosis in $75 \%$ of patients, identifying acute myocarditis, myocardial infarction with spontaneous recanalisation/embolus, and cardiomyopathies. Establishing a final diagnosis has an important impact in patient management and secondary prevention.

\section{CHRONIC DPP-4 INHIBITION BY SITAGLIPTIN ENHANCES BOTH GLOBAL AND REGIONAL MYOCARDIAL FUNCTION DURING DOBUTAMINE STRESS IN PATIENTS WITH TYPE 2 DIABETES MELLITUS AND CORONARY ARTERY DISEASE}

doi:10.1136/heartjnl-2012-301877b.135

L M McCormick,* A C Kydd, P A Read, S P Hoole, D P Dutka. Department of Cardiovascular Medicine, University of Cambridge, Cambridge, UK

Background Glucagon-like peptide-1 (GLP-1) is an incretin hormone secreted by the gut in response to the oral ingestion of nutrients. Through augmentation of myocardial glucose uptake and subsequent reduction in fatty acid oxidation, GLP-1 modulation therapy has emerged as a potential target for improving myocardial oxygen efficiency at times of ischaemic stress, such as occurs in obstructive coronary artery disease (CAD). Sitagliptin is a DDP-4 inhibitor licensed for the treatment of Type 2 Diabetes Mellitus (T2DM) that reduces degradation of plasma GLP-1 (7-36). We investigated whether sitagliptin improved myocardial performance during dobutamine stress echocardiography (DSE) in patients with T2DM and $\mathrm{CAD}$

Methods 12 patients (aged 69 \pm 9 years, 9 men) with T2DM on oral hypoglycaemic therapy (OHT), obstructive $\mathrm{CAD}$ and preserved left ventricular (LV) systolic function were studied. Each subject underwent DSE on two separate occasions after an overnight fast; the first (control) while receiving standard OHT and the second after the addition of sitagliptin (100 mg od) for 4 weeks. Tissue Doppler imaging was acquired in three apical views at rest, peak stress and 30 min recovery. Global function was assessed by ejection fraction (EF) using the Simpson's biplane method and mitral annular peak systolic velocity (MASV) averaged over six sites. Regional LV wall motion was assessed using a 12-segment model comprising the base and mid-level of six regional walls. Peak systolic tissue velocity (Vs), strain (S) and strain rate (SR) were calculated for each region from tissue Doppler velocity data averaged over three consecutive beats using an off-line workstation (EchoPac, GE Medical Systems).

Results At rest, all parameters of both global and regional LV performance were unchanged after sitagliptin. At peak stress, the rate-pressure product attained was the same for both DSE studies but those after sitagliptin demonstrated significantly enhanced myocardial performance in both global (LVEF $69.9 \pm 6.5$ vs $63.9 \pm 6.2 \%, \quad p=0.001 ;$ MASV $12.69 \pm 3.0$ vs $11.65 \pm 3.5 \mathrm{~cm} / \mathrm{s}$, 